\title{
Train the Vessel, Gain the Brain: Physical Activity and Vessel Function and the Impact on Stroke Prevention and Outcome in Cerebrovascular Disease
}

\author{
Wolf Schmidt ${ }^{\mathrm{a}, \mathrm{b}}$ Matthias Endres ${ }^{\mathrm{a}-\mathrm{c}}$ Fernando Dimeo $^{\mathrm{d}}$ \\ Gerhard J. Jungehulsing ${ }^{a, b}$ \\ ${ }^{a}$ Center for Stroke Research Berlin (CSB), ${ }^{b}$ Department of Neurology, ${ }^{c}$ Cluster of Excellence NeuroCure and \\ dSection of Sports Medicine, Department of Oncology at Campus Benjamin Franklin, Charité University \\ Medicine Berlin, Berlin, Germany
}

\section{Key Words}

Physical activity · Cerebral blood flow · Neovascularization .

Angiogenesis · Arteriogenesis · Neurogenesis

\begin{abstract}
The burden of cerebrovascular disease (CVD) is huge and therapeutic options are limited. Physical activity is effective in preventing coronary heart and peripheral artery disease both experimentally and clinically. It is likely that the protective effects of exercise can be extended to both CVD and cognitive impairment. The pleiotropic protective and preventive mechanisms induced by physical activity include increased perfusion as well as mechanisms of collateral recruitment and neovascularization mediated by arterio- and angiogenesis. Physical activity increases the bioavailability of nitric oxide, bone marrow-derived CD34+ cells and growth factors, all of which promote neovascularization. Additionally, shear stress is discussed as a potential mechanism for vessel growth. Moreover, physical activity plays a role in endothelial function and cerebral autoregulation in small- and large-artery CVD. The vascular niche hypothesis highlights the complex interactions of neuro- and angiogenesis for regenerative and repair mechanisms in the human brain. Ex-
\end{abstract}

perimental and clinical studies demonstrate the positive impact of prior physical activity on stroke lesion size and on outcome after stroke. Clinical trials are necessary to further address the impact of physical activity on primary and secondary stroke prevention, outcome and cognitive function.

Copyright $\odot 2013$ S. Karger AG, Basel

\section{Introduction}

Physical activity reduces cardiovascular morbidity and mortality by modulation of 'classical' risk factors such as hypertension, diabetes and dyslipidemia $[1,2]$ (fig. 1). Shown to improve endothelial function in peripheral (PAD) and coronary artery disease (CAD) $[3,4]$, the effects of physical activity are likely mediated by increased bioavailability of nitric oxide (NO) and consecutive endothelium-dependent vasodilatation [5-7]. Moreover, exercise is a strong stimulus promoting neovascularization including arterio- and angiogenesis. Most pleiotropic effects of regular physical activity have been investigated either in healthy individuals or in PAD and CAD patients. So far, there is limited but growing evidence that physical activity might also play a preventive

\section{KARGER}

E-Mail karger@karger.com

www.karger.com/ced (c) 2013 S. Karger AG, Basel

$1015-9770 / 13 / 0354-0303 \$ 38.00 / 0$
Wolf Schmidt, MD, MS

Center for Stroke Research Berlin (CSB) and Department of Neurology

Charité University Medicine Berlin

Charité-Platz 1, DE-10117 Berlin (Germany)

E-Mail wolf.schmidt@ charite.de 


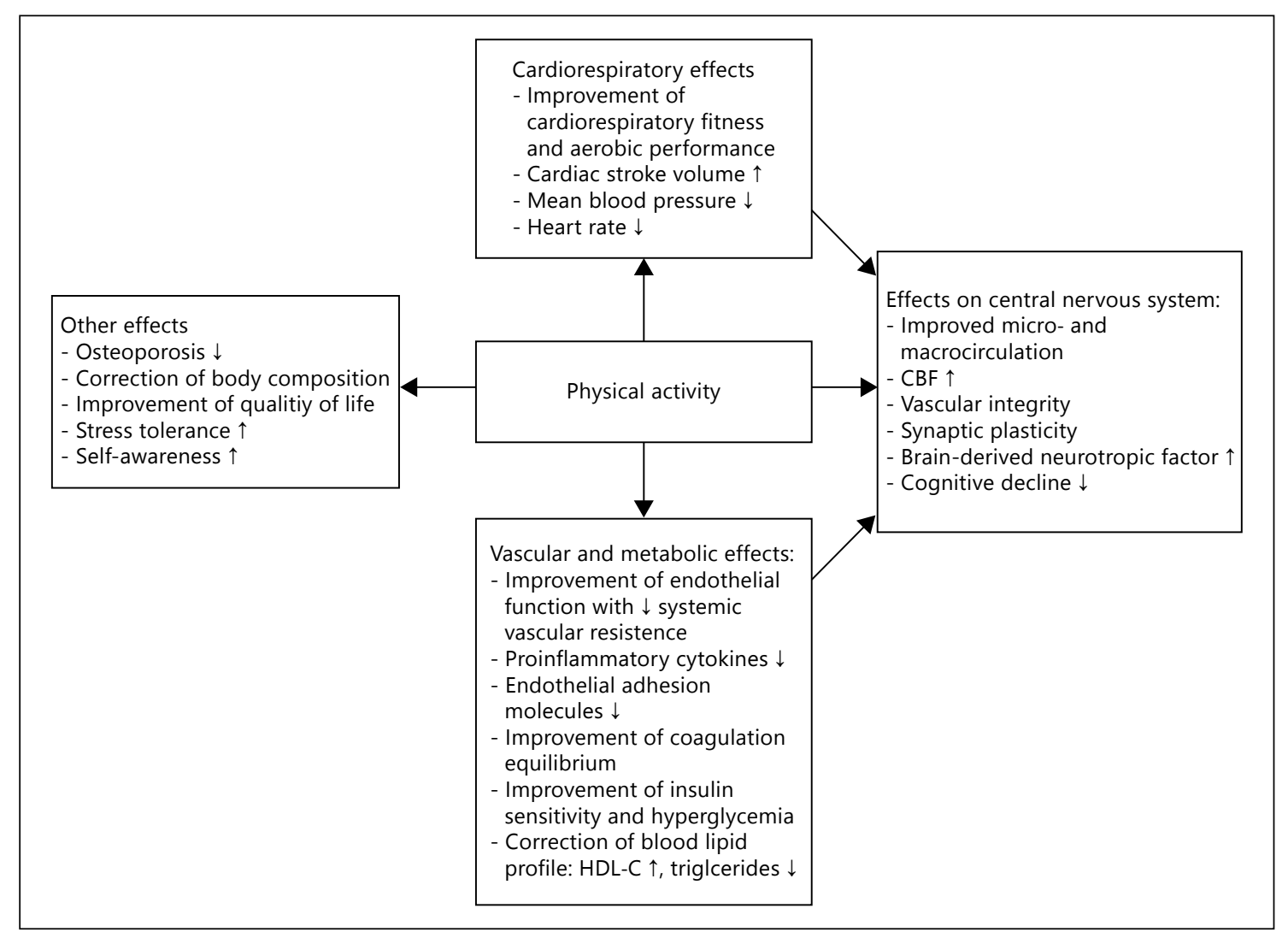

Fig. 1. Pleiotropic effects of exercise and modulated cardiovascular risk factors [modified from 2].

role in small- and large-artery cerebrovascular disease (CVD) as well as in vascular and neurodegenerative dementia $[8,9]$.

In this review we will highlight the relationship between physical activity and CVD. We aim to illustrate the influence of exercise on endothelial function and neovascularization prior to stroke, the principles of collateral recruitment and the effects of exercise on cerebral hemodynamics. We will give an overview on the impact of exercise on stroke prevention and outcome and the influence of exercise on cognitive function. Finally, we will provide an outlook on future developments in research and on possible therapeutic options based on the vascular niche hypothesis.

\section{Neovascularization in the Human Brain and Physical Activity}

Various vascular mechanisms have been proposed to explain how exercise influences the maintenance, augmentation and/or improvement of cerebral blood flow
$(\mathrm{CBF})$ and perfusion. All may crucially exert beneficial effects on stroke prevention and healthy human aging and are due to physical activity. The mechanisms include collateral recruitment and arterio-, vasculo- and angiogenesis.

\section{$\mathrm{CBF}$ and Collateral Recruitment}

In individuals with a functional stenosis of a large brain-supplying artery, cerebral autoregulation and regional CBF are often impaired [10]. Cerebral autoregulation is an essential intrinsic property in maintaining a constant $\mathrm{CBF}$ despite changes in cerebral perfusion pressure. $\mathrm{CBF}$ is kept constant in a range thought to be 50-60 $\mathrm{ml} / 100 \mathrm{~g} / \mathrm{min}[11]$ despite changes in mean arterial pressure by mechanoregulatory (blood pressure regulation) and chemoregulatory $\left(\mathrm{PaCO}_{2}\right)$ compensatory mechanisms, both leading to a myogenic response resulting either in vasodilatation or the constriction of smooth muscle cells in cerebral arteries and arterioles.

The extent of impairment of the cerebral autoregulation depends among other factors upon the degree of cerebral collateralization [10], which in turn also influences 
the cerebrovascular reserve capacity (CRC) $[12,13]$. The CRC refers to the brain's capacity to increase cerebral blood volume (CBV) and maintain a constant regional $\mathrm{CBF}$ in the face of low cerebral perfusion pressure. This can be quantified by PET, SPECT or Doppler ultrasound. It provides information as to whether arterioles are already maximally dilated (to maintain blood flow and metabolic needs in the poststenotic area) or if response to an increased demand is possible, for example, due to decreases in blood pressure [14]. A severely impaired CRC is associated with an increased risk of stroke or transient ischemic attack (TIA) in patients with high-grade carotid artery stenosis or occlusion [15].

The recruitment of existing collateral vessels within the circle of Willis is an endogenous protective mechanism by which sufficient blood supply can be maintained in $80 \%$ of all patients with high-grade extra- or intracranial stenosis or occlusion of brain-supplying arteries [for review, see 16]. For example, if unilateral carotid occlusion with a decrease in perfusion pressure occurs, interhemispheric 'cross-flow' via the anterior communicating artery may develop [17]. This is then associated with a reduced risk of internal hemodynamic watershed infarctions $[18,19]$. Primary anastomoses are dependent upon the anatomic configuration of the circle of Willis. Consequently, the number of existing collaterals is inversely correlated with stroke risk [13]. Secondary collaterals independent of the circle of Willis develop under conditions of chronic ischemia or peripheral vessel occlusions via anastomoses of the internal and external carotid arteries, such as the ophthalmic artery or leptomeningeal arteries between the three intracranial vascular territories [16]. The extent of collateral circulation and the vasodilatory ability of the vasculature determine the CRC [12]. Exercise might improve CRC by inducing neovascular processes and, therefore, influence the risk of stroke or TIA (http://clinicaltrials.gov/ct2/show/NCT00912561).

Arteriogenesis - Outward Growth of Collateral Vessels The adaptive reaction to an increase in diameter of collateral vessels in large-artery occlusive disease is referred to as arteriogenesis [20]. In rat models, the complex vascular mechanisms of arteriogenesis can be accelerated by different cyto- and chemokines. Several coronary and peripheral models of arteriogenesis have been developed to study the mechanisms that occur in preexisting arteriolar anastomosis-inducing mitosis in endothelial and smooth muscle cells [21]. Relevant cyto- and chemokines include monocyte chemoattractant protein 1 (MCP-1), granulocyte macrophage colony-stimulating factor (GM-CSF),

Physical Activity and Vessel Function in CVD fibroblast growth factor-beta, and vascular endothelial growth factor (VEGF), which also modulate arteriogenesis in the brain [20,22-24].

Though stimulation of arteriogenesis by cytokines is promising in animal models, randomized controlled trials in humans with application of cytokines as fibroblast growth factor $[25,26]$ or VEGF $[27,28]$ failed to improve clinical endpoints (e.g. angina score and walking distance in patients with CAD or PAD). Application of GM-CSF improved coronary collateralization as measured by invasive collateral flow index in CAD patients [29]. However, the START trial failed to demonstrate an improvement of the walking distance in PAD patients [30]. Additionally, in patients with stable $\mathrm{CAD}$, a higher incidence of acute coronary syndrome was seen after subcutaneous application of GM-CSF [31]. The AXIS-1 trial demonstrated safety and tolerability of different dosages of GCSF in patients with acute ischemic stroke but just recently it was reported that the phase II clinical trial (AXIS2) with the G-CSF compound AX200 missed all primary and secondary endpoints [32].

Finally, arteriogenesis might be 'mechanically' stimulated by fluid shear stress due to an exercise-induced increase of CBF. Schierling et al. [33] demonstrated in a murine cerebral 3-vessel occlusion model that cerebral arteriogenesis is dependent on fluid shear stress. It is directly augmented by an increased blood flow due to an increase in the tangential forces exerted by the blood on the endothelial lining [34]. This exertion upon the endothelium then initiates a consecutive cascade of endothelial mechanisms including: (1) the expression of adhesion molecules like intracellular adhesion molecule (ICAM)-1 and (2) the upregulation of the endothelial NO synthase (eNOS) system and MCP-1. This in turn triggers an invasion of bone marrow-derived mononuclear cells and creates a complex cytokine environment and consecutive proliferation of endothelial and smooth muscle cells [34] (see fig. 2). In conclusion, arteriogenesis is a highly complex process based on a sophisticated interplay of different cytokines, enzymes, growth factors, cell types and populations, and direct stimulation of arteriogenesis might be a therapeutic approach in occlusive artery diseases [for review, see 35].

\section{Angiogenesis, Postnatal Vasculogenesis and the Role of Endothelial Progenitor Cells}

Angiogenesis (the sprouting of new capillaries from preexisting blood vessels) is thought to be the primary mechanism of new vessel formation in the adult [36]. In contrast, postnatal vasculogenesis signifies the forma- 
Fig. 2. Angiogenesis, arteriogenesis and neurogenesis: exercise induces liberation of bone marrow-derived stem cells (stage A) circulating into the periphery. This is highly NO-dependent and caused by an upregulation of eNOS in stromal cells and leads to MMP-9 activation but is also enhanced by VEGF, angiopoietin-1, fibroblast growth factor, GM-CSF and pharmacological agents such as statins and erythropoietin $[47,50]$. Exercise-induced shear stress (arrows, stage B) causes a cascade of mechanisms including the expression of intracellular adhesion molecule (ICAM)-1 and the upregulation of the eNOS system and MCP-1. This, in turn, triggers an invasion of bone marrow-derived mononuclear cells creating a complex cytokine environment and consecutive proliferation of endothelial and smooth muscle cells leading to an increase in diameter of an existing collateral artery (arteriogenesis) [34]. On a capillary level, EPCs are involved in the sprouting of existing capillaries and by the release of BDNF and VEGF (stage C), resulting in either recruitment of resident latent neuronal progenitors or neuroblast migration, thus facilitating neurogenesis.

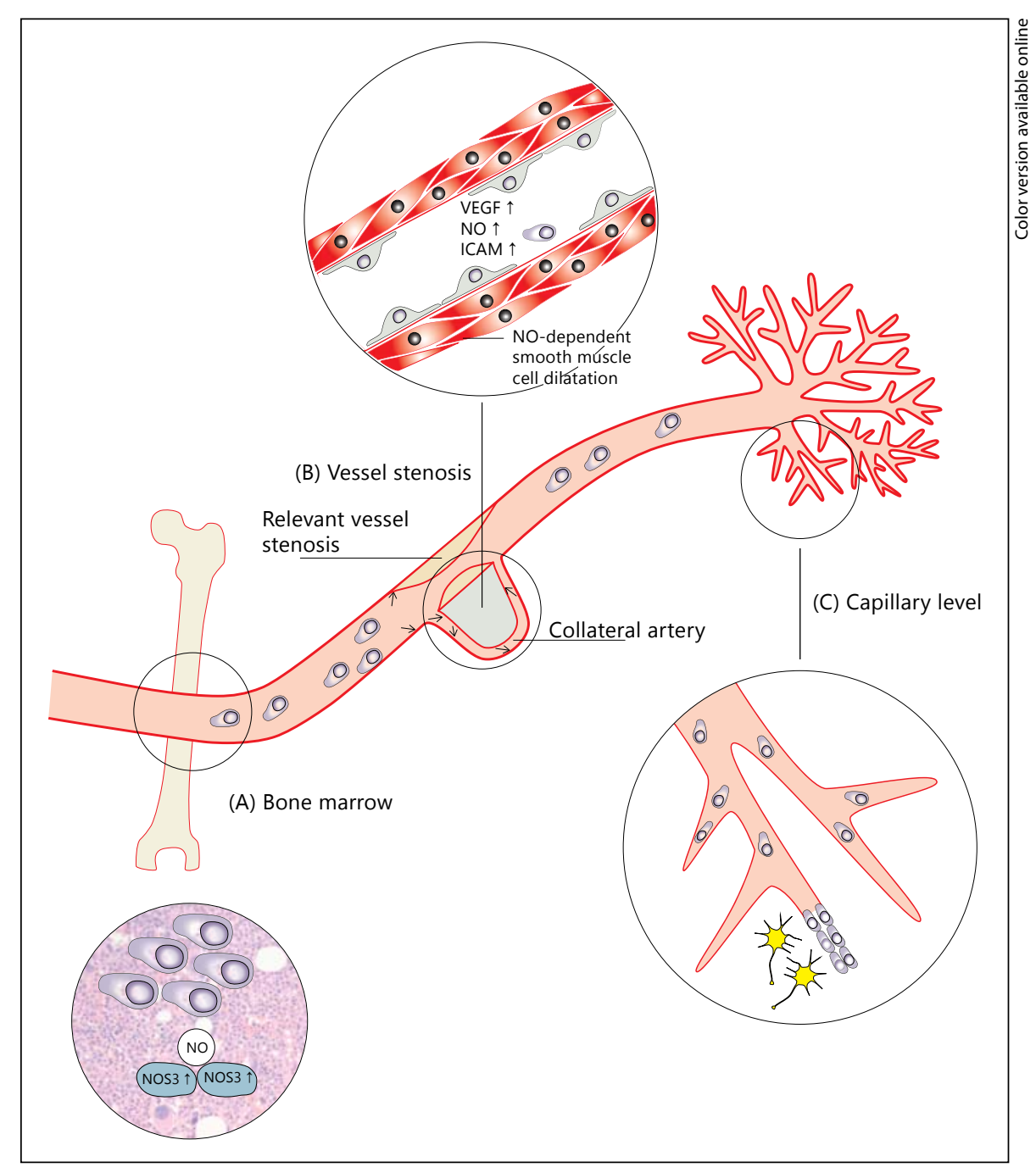

tion of new vessels from endothelial progenitor cells (EPCs) [36]. While monocytes are considered prerequisites in the process of arteriogenesis [20], EPCs play an important role for angio- and vasculogenesis [37]. EPCs are bone marrow-derived stem cells and are distinct from mature endothelial cells in that they possess the potential to differentiate into endothelial cells. The definition of EPCs remains controversial [38]. In general, there are two main techniques to assess EPCs: flow cytometry (fluorescence-activated cell sorting, FACS) and in vitro assays as cell cultures. While FACS allows a detailed characterization of naive cells and different subpopulations, the technique is complex and susceptible to falsepositive events, and a functional evaluation of the cell population is not possible. In contrast, cell cultures allow a functional evaluation of larger quantities of cultured cells but, owing to the necessary culture medium supply and the unphysiological condition of cell cultures, physiological properties of these manipulated cells might be concealed. Usually, EPCs are phenotyped by a combination of markers specific for stem and endothelial cells with FACS. For example, typical combinations include the hematopoietic surface marker CD34 for hematopoietic cells, CD133 as a stem cell and VEGF receptor 2 (CD309) as an endothelial marker [39]. CD133 expression seems to be restricted to early EPCs and the surface antigen is lost during maturation to endothelial cells, which allows an identification of the maturation states of EPCs [40-42]. The number of EPCs seems to be associated with endothelial dysfunction [43] and has been found to be a better predictive risk factor for cardiovascular events than the combined Framingham score, un- 
derlining the putative role of EPCs in vascular homeostasis and repair [44]. In the EPCAD study, the cumulative event survival increased stepwise across baseline EPC levels for the occurrence of a first major cardiovascular event [45]. Increased CD34+ counts have been found in patients with stenosis of carotid or middle cerebral artery and accelerated neovascularization characterized by moyamoya-like vessels. This has led to the conclusion that increased levels of bone marrow-derived CD34+ cells are correlated with neovascularization of the cerebral arterial circulation [46]. Therefore, EPCs might play an essential role in vascular regeneration, development and collateralization of the human brain. Even short-term exercise increases the number of circulating EPCs $[47,48]$ and a 4-week exercise program resulted in a lasting increment of circulating EPCs [49]. Exercise training also induces qualitative properties of EPCs by increasing expression of homing factor CXCR4, which might promote an improved integration of EPCs into endothelial networks [50].

\section{The 'Vascular Niche of Neurogenesis' Hypothesis}

According to the vascular niche hypothesis, regeneration and repair mechanisms can only proceed by adult neurogenesis in an angiogenic environment [51]. Adult neurogenesis is the process of generating new neurons that integrate into exiting neuronal networks after fetal and early postnatal neurogenesis has ceased [52]. The findings from both experimental stroke and dementia studies underline the importance of a comprehensive consideration of the complex interactions between brain vasculature and neuronal function within the neurovascular unit [52]. Also antiangiogenic approaches in cancer contributed to the knowledge of mechanisms in the neurovascular unit, as recently reviewed by Carmeliet and Jain [53].

Endothelial cells release neurotrophic factors like brain-derived neurotrophic factor (BDNF) and VEGF [54], which stimulate the renewal of adult neuronal stem and progenitor cells and promote the production of neurons. In the adult brain, neurons and glia cells are generated throughout life in the subventricular zone, the posterior periventricular area and the subgranular zone of dentate gyrus in vitro and in the adult human brain $[55,56]$. Available data in rodents show that under physiological conditions, neuroblasts from the subventricular zone migrate to the olfactory bulb [57]. Under ischemic conditions, neuroblasts migrate towards the ischemic boundary, where angiogenesis occurs and neuroblasts and cerebral vessels are linked together [58].
Endothelial cells also promote proliferation of neural progenitor cells and neuronal differentiation. Reciprocally, neural progenitor cells promote angiogenesis in vitro and VEGF seems to be a main growth factor facilitating this coupling [59], which also seems to occur in the human adult. Pereira et al. [60] showed in an MRI study that exercise increases CBV in the adult human dentate gyrus where exercise-induced neuro- and angiogenesis have been demonstrated in animals [37, 6163].

Increased cortical CBV is correlated with angiogenesis in ischemia [64] and neoplastic processes such as gliomas [65]. In turn angiogenesis occurs in the vascular/neurogenic niche of the hippocampus [51]. Because of the correlation between CBV/angiogenesis and angiogenesis/ neurogenesis, CBV measurement might be an indirect parameter for neurogenesis.

To conclude, the vascular niche hypothesis gives insights into the complex interactions of neuro- and angiogenesis [for review see 66] that can be influenced and stimulated by regular physical exercise. Exercise does not only strengthen muscle, it also strengthens the cerebral vasculoneuronal network.

\section{Impact of Physical Activity on Stroke Outcome}

\section{Preclinical Evidence}

Exercise has been shown to reduce infarction size and neurological deficits and to ameliorate brain injury after focal cerebral ischemia in a murine middle cerebral artery occlusion model [7]. This is attributed to increased expression and activity of eNOS, NO-dependent vasodilatation and regional CBF [7]. In addition, exercise inhibits injury due to inflammation by decreasing ICAM-1 expression and consecutive lower leukocyte accumulation in damaged brain and causes an overexpression of neutrophils as BDNF in rats [67]. Early exercise after stroke might also be beneficial [68]. A subsequent study in a murine model revealed that voluntary exercise prior to acute cerebral ischemia increased levels of VEGF, which activates eNOS. Furthermore, exercise augmented the recruitment of EPCs into the ischemic region and increased the microvascular density and CBF both by angiogenesis and vasorelaxation, improving long-term recovery and regeneration $[37,69]$. In conclusion, exercise promotes short- and long-term effects that increase cerebral perfusion and functional outcome by activation and expression of eNOS and EPCs after experimental ischemia in the mouse model. 


\section{Clinical Evidence}

Individuals who were physically active prior to stroke have been shown to have less severe strokes and an improved stroke outcome [70, 71]. Light-to-moderate leisuretime activity was associated with an odds ratio of 4.18 (CI 1.55-11.26) for a lower severity of stroke upon hospital admission in 362 patients [70]. In addition, severity of stroke was lower in moderately active stroke patients, even though stroke size did not differ between low, moderate or high leisure-time physical activity levels [71]. Moreover, higher prestroke levels of physical activity are associated with an increased likelihood of good short-term (within 8 days) [70] and long-term (up to 2 years) [72] outcomes, measured by the NIHSS and modified Rankin Scale, respectively.

\section{Exercise and Cognitive Function}

The effects of exercise on healthy ageing and maintenance of cognitive function have been analyzed in a number of studies [73]. Cognitive impairment occurs more frequently in CVD patients than in age-matched controls [74]. It has been shown that endothelial dysfunction is associated with a high lesion load of white matter hyperintensities in MRI, underlining the importance of endothelial integrity and vasomotion in CVD [75]. There is a growing body of evidence suggesting that vascular and neurodegenerative dementias have common features that are crucially dependent upon the regulation of $\mathrm{CBF}$ on a microcirculatory level [76] and that exercise exerts beneficial effects on neurodegenerative dementia, vascular dementia and mild cognitive impairment $[77,78]$.

It is a matter of debate as to whether exercise might improve cognitive function in patients without known cognitive impairment [79]. It is known, however, that a sedentary lifestyle has a negative effect on neuronal plasticity and learning [80] and that aerobic fitness reduces brain tissue loss [81]. There are several hypotheses as to how exercise could influence executive control and alertness. First, exercise increases CBV [60] and CBF [7] - including in the dentate gyrus and hippocampus [82]. Second, the so-called 'arousal hypothesis' suggests that exercise induces an increase of catecholamines $[83,84]$ and thus increases arousal $[85,86]$. Third, it is known that exercise upregulates neutrophils, in particular BDNF [87], facilitating dendritic branching and synaptic plasticity [88] (fig. 2). Flöel et al. [89] and Ruscheweyh et al. [90] showed that individuals with higher levels of physical activity had a better memory function and higher levels of neutrophils than G-CSF and BDNF, as well as increased gray matter volume in cingulate and prefrontal areas. Finally, exercise improves mood and alleviates depression, which may in turn also affect cognitive function [91, 92].

During normal healthy aging CBF declines up to $50 \%$ [93-95] while cerebral autoregulation seems to be preserved [96]. Because no changes in regional CBF have been detected, it is assumed that this decrease reflects a global perfusion decrease associated with cerebral atrophy. Interestingly, Ainslie et al. [95] observed an increase of $\mathrm{CBF}$ velocity of the middle cerebral artery in endurance-trained men compared to individuals with a sedentary life style. This increase was persistent in all age groups and confirms the results of exercise-induced CBF increase in animal studies $[7,37]$. One might speculate that this increase of $\mathrm{CBF}$ velocity reflects the lower cerebrovascular risk in physically active elderly as well as a protective mechanism against cerebral atrophy.

\section{Physical Exercise in Primary Prevention of CVD}

Several epidemiological cohort and case-control studies have addressed whether regular physical activity reduces the risk of stroke. Observational studies found associations between physical activity and stroke risk. The Honolulu Heart Study found that regular physical activity protected against hemorrhagic stroke incidence after examining over 7,500 Asian men for a 20-year period [97]. In the Oslo Study, leisure-time physical activity reduced the relative risk of stroke morbidity and mortality in middle-aged men [98]. Likewise, the incidence of all stroke subtypes was lower in physically active woman between the ages of 40 and 65 in the Nurses' Health Study [99] and also in the Women's Health Study [100], while women with low recreational physical activity showed a higher relative risk of stroke [101]. The Northern Manhattan Stroke Study found a protective effect of leisure-time activity (adjusted for vascular risk factors) for all stroke subtypes in Blacks, Whites and Hispanics over all age groups [102].

\section{The Role of Exercise Intensity}

It is not known whether there is an optimum of exercise intensity or a dose-response correlation of physical activity for stroke prevention. Linear [99, 103, 104], U-shaped $[105,106]$ and constant correlations for all activity levels [107] have been described. Two recent meta-analyses investigated the association of physical activity and stroke risk stratified by grade of activity $[108,109]$. Both studies came to the conclusion that moderately intense exercise is sufficient to achieve a risk reduction for total stroke and 
stroke subtypes. However, the influence of intensities or frequencies of exercise have rarely been investigated. Selfreported frequency and intensity with individual outcome measures such as sweat production [110] and dichotomization (active vs. inactive) or trichotomization (self-reported habitual low, moderate or high physical activity) [101] both in observational epidemiological and case-control studies limit the comparability between different studies. In British middle-aged men, the risk of heart attack was slightly increased in vigorously exercising men compared to those training at a moderate or moderate-to-vigorous level once weekly [111]. Furthermore, there is some evidence that frequent vigorous exercise is associated with an increased risk of atrial fibrillation [112], while light-tomoderate leisure-time activity and walking are associated with significantly lower atrial fibrillation incidence in older adults [113]. Recent data from the Northern Manhattan Stoke study suggest that moderate-to-high intensity physical activity was associated with a lower risk of ischemic stroke in men [114]. Grau et al. [115] pointed out that not only lifetime physical activity is associated with reduced odds of stroke or TIA. In that study, recently initiated exercise in individuals without a history of physical activity in young adulthood was also associated with reduced odds of stroke and TIA. Most patients exercised more than 2 but less than $7 \mathrm{~h}$ per week. The effects of training as they pertain to secondary stroke prevention, death, dependence and disability after stroke remain unclear [116]. Although secondary prevention programs for stroke patients exist, they are not as well studied or widely available as secondary prevention programs for cardiac patients [117].

Most clinical studies investigate the impact of endurance exercise activities such as cycling, walking or jogging on cardiovascular and stroke prevention. However, it was shown that even moderate-intensity resistance training is as effective as endurance sport for the prevention and treatment of high blood pressure [118]. Exercise and rehabilitation protocols tailored for specific stroke deficits in stroke survivors differ. Such training protocols mostly aim to improve the neurological motor deficit, but in addition it is shown that exercise in stroke survivors reduces stroke severity and improves stroke outcome [70-72].

\section{Summary and Conclusions}

There is growing evidence that physical activity prevents CVD and reduces cerebro- and cardiovascular morbidity and mortality. Exercise prior to stroke might improve stroke outcome and has been shown to improve

Physical Activity and Vessel Function in CVD cognitive impairment in vascular and degenerative dementia. In contrast, secondary stroke prevention data are missing. The beneficial effects of exercise are multifaceted. First, exercise modulates cardiovascular risk factors. Second, exercise plays a role in the mediation of vascular and other endothelial effects. Apart from recruiting collateral vessels, physical activity can improve vessel collateralization by augmented arterio- and/or angiogenesis. The underlying physiological mechanisms for improved angiogenesis can be attributed to an increased phosphorylation of eNOS, elevated levels of bone marrow-derived CD34+ cells (e.g. EPCs) and subsequent endothelial or mechanical mechanisms leading to neovascularization and correction of endothelial dysfunction. According to the vascular niche hypothesis, neuronal regeneration is enhanced in the angiogenic environment - probably not only in the ischemic brain. No dedicated recommendations on the timing or dosage of physical activity can be given, but further randomized controlled trials should be encouraged.

In our view such trials should focus on the subacute phase weeks after stroke because the potential benefit of exercise in this phase can be assumed to be highest. Such trials should also investigate the right type and dosage of exercise intervention and the different levels of outcome (e.g. depression, activities of daily living) after an exercise intervention, as well as the surrogate parameters (e.g. $\mathrm{CBF}$, endothelial markers) of the potential underlying vascular mechanisms.

However, in the end there is no doubt that, in general, exercise can be recommended as a nonpharmacological and noninvasive intervention in the primary and secondary prevention of stroke for the sustainment of cognitive function, and finally for the improvement of outcome and rehabilitation after stroke. Strain the muscles, train the vessels, and gain the brain!

\section{Acknowledgements}

The authors (W.S. and G.J.J.) acknowledge financial support from the Federal Ministry of Education and Research via the grant Center for Stroke Research Berlin (01 EO 0801). M.E. receives funding from the DFG (Excellence cluster NeuroCure; SFB TR 43, KFO 247, KFO 213), BMBF (Centre for Stroke Research Berlin), EU (Eustroke, ARISE, WakeUp), Volkswagen Foundation (Lichtenberg Program), Corona Foundation.

\section{Disclosure Statement}

The authors report no conflicts of interest. 


\section{References}

$>1$ Physical activity and cardiovascular health. $>18$ Bisschops RH, et al: Collateral flow and ischNIH Consensus Development Panel on Physical Activity and Cardiovascular Health. JAMA 1996;276:241-246.

2 Bluher M, Zimmer P: Metabolic and cardiovascular effects of physical activity, exercise and fitness in patients with type 2 diabetes (in German). Dtsch Med Wochenschr 2010;135: 930-934.

$\checkmark 3$ Walther C, Gielen S, Hambrecht R: The effect of exercise training on endothelial function in cardiovascular disease in humans. Exerc Sport Sci Rev 2004;32:129-134.

$\checkmark 4$ Maiorana A, et al: Exercise and the nitric oxide vasodilator system. Sports Med 2003;33: 1013-1035.

5 Hambrecht R, et al: Regular physical activity improves endothelial function in patients with coronary artery disease by increasing phosphorylation of endothelial nitric oxide synthase. Circulation 2003;107:3152-3158.

$\checkmark 6$ Hambrecht R, et al: Regular physical exercise corrects endothelial dysfunction and improves exercise capacity in patients with chronic heart failure. Circulation 1998;98: 2709-2715.

7 Endres M, et al: Mechanisms of stroke protection by physical activity. Ann Neurol 2003;54: 582-590.

$>8$ Rolland Y, van Kan GA, Vellas B: Healthy brain aging: role of exercise and physical activity. Clin Geriatr Med 2010;26:75-87.

$>9$ Leung FP, et al: Exercise, vascular wall and cardiovascular diseases: an update. Part 1. Sports Med 2008;38:1009-1024.

$>10$ Reinhard, M, et al: Dynamic cerebral autoregulation and collateral flow patterns in patients with severe carotid stenosis or occlusion. Ultrasound Med Biol 2003;29:1105-1113.

11 Lassen NA: Cerebral blood flow and oxygen consumption in man. Physiol Rev 1959;39: 183-238.

12 Ozgur HT, et al: Correlation of cerebrovascular reserve as measured by acetazolamidechallenged SPECT with angiographic flow patterns and intra- or extracranial arterial stenosis. AJNR Am J Neuroradiol 2001;22:928936.

13 Vernieri F, et al: Effect of collateral blood flow and cerebral vasomotor reactivity on the outcome of carotid artery occlusion. Stroke 2001; 32:1552-1558.

14 Widder B, Kleiser B, Krapf H: Course of cerebrovascular reactivity in patients with carotid artery occlusions. Stroke 1994;25:1963-1967.

$\checkmark 15$ Markus H, Cullinane M: Severely impaired cerebrovascular reactivity predicts stroke and TIA risk in patients with carotid artery stenosis and occlusion. Brain 2001;124:457-467.

16 Liebeskind DS: Collateral circulation. Stroke 2003;34:2279-2284.

17 Lindegaard KF, et al: Assessment of intracranial hemodynamics in carotid artery disease by transcranial Doppler ultrasound. J Neurosurg 1985;63:890-898. emic brain lesions in patients with unilatera carotid artery occlusion. Neurology 2003;60: 1435-1441.

19 Hendrikse J, Eikelboom BC, van der Grond J: Magnetic resonance angiography of collateral compensation in asymptomatic and symptomatic internal carotid artery stenosis. J V asc Surg 2002;36:799-805.

20 Arras M, et al: Monocyte activation in angiogenesis and collateral growth in the rabbit hindlimb. J Clin Invest 1998;101:40-50.

21 Schaper W, De Brabander M, Lewi P: DNA synthesis and mitoses in coronary collateral vessels of the dog. Circ Res 1971;28:671-679.

22 Busch HJ, et al: Arteriogenesis in hypoperfused rat brain. J Cereb Blood Flow Metab 2003;23:621-628.

23 Buschmann IR, et al: Therapeutic induction of arteriogenesis in hypoperfused rat brain via granulocyte-macrophage colony-stimulating factor. Circulation 2003;108:610-615.

24 Hoefer IE, et al: Time course of arteriogenesis following femoral artery occlusion in the rab bit. Cardiovasc Res 2001;49:609-617.

25 Grines CL, et al: Angiogenic Gene Therapy (AGENT) Trial in patients with stable angina pectoris. Circulation 2002;105:1291-1297.

$>26$ Simons M, et al: Pharmacological treatment of coronary artery disease with recombinant fibroblast growth factor-2: double-blind, randomized, controlled clinical trial. Circulation 2002;105:788-793.

27 Henry TD, et al: The VIVA Trial: vascular endothelial growth factor in ischemia for vascular angiogenesis. Circulation 2003;107:13591365.

28 Rajagopalan S, et al: Regional angiogenesis with vascular endothelial growth factor in pe ripheral arterial disease: a phase II randomized, double-blind, controlled study of adenoviral delivery of vascular endothelial growth factor 121 in patients with disabling intermittent claudication. Circulation 2003;108:19331938.

29 Seiler C, et al: Promotion of collateral growth by granulocyte-macrophage colony-stimulating factor in patients with coronary artery disease: a randomized, double-blind, placebocontrolled study. Circulation 2001;104:20122017.

30 van Royen N, et al: START Trial: a pilot study on stimulation of arteriogenesis using subcutaneous application of granulocyte-macrophage colony-stimulating factor as a new treatment for peripheral vascular disease. Circulation 2005;112:1040-1046.

31 Zbinden S, et al: Safety and efficacy of subcutaneous-only granulocyte-macrophage colony-stimulating factor for collateral growth promotion in patients with coronary artery disease. J Am Coll Cardiol 2005;46:16361642.
32 Schabitz WR, et al: AXIS: a trial of intravenous granulocyte colony-stimulating factor in acute ischemic stroke. Stroke 2010;41: 2545-2551.

33 Schierling W, et al: Increased intravascular flow rate triggers cerebral arteriogenesis. J Cereb Blood Flow Metab 2009;29:726-737.

34 Heil M, Schaper W: Influence of mechanical, cellular, and molecular factors on collateral artery growth (arteriogenesis). Circ Res 2004; 95:449-458.

35 Schaper W: Collateral circulation: past and present. Basic Res Cardiol 2009;104:5-21.

36 Risau W: Mechanisms of angiogenesis. Nature 1997;386:671-674.

37 Gertz K, et al: Physical activity improves longterm stroke outcome via endothelial nitric oxide synthase-dependent augmentation of neovascularization and cerebral blood flow. Circ Res 2006;99:1132-1140.

38 Li Calzi S, et al: EPCs and pathological angiogenesis: when good cells go bad. Microvasc Res 2010;79:207-216.

39 Shantsila E, Watson T, Lip GY: Endothelial progenitor cells in cardiovascular disorders. J Am Coll Cardiol 2007;49:741-752.

40 Hristov M, Weber C: Endothelial progenitor cells: characterization, pathophysiology, and possible clinical relevance. J Cell Mol Med 2004;8:498-508.

41 Peichev M, et al: Expression of VEGFR-2 and AC133 by circulating human CD34(+) cells identifies a population of functional endothelial precursors. Blood 2000;95:952-958.

42 Rafii S, Lyden D: Therapeutic stem and progenitor cell transplantation for organ vascularization and regeneration. Nat Med 2003;9: 702-712.

43 Werner N, et al: Endothelial progenitor cells correlate with endothelial function in patients with coronary artery disease. Basic Res Cardiol 2007;102:565-571.

44 Hill JM, et al: Circulating endothelial progenitor cells, vascular function, and cardiovascular risk. N Engl J Med 2003;348:593-600.

45 Werner N, et al: Circulating endothelial progenitor cells and cardiovascular outcomes. $\mathrm{N}$ Engl J Med 2005;353:999-1007.

$\checkmark 46$ Yoshihara $\mathrm{T}$, et al: Increase in circulating CD34-positive cells in patients with angiographic evidence of moyamoya-like vessels. J Cereb Blood Flow Metab 2008;28:1086-1089.

47 Adams V, et al: Increase of circulating endothelial progenitor cells in patients with coronary artery disease after exercise-induced ischemia. Arterioscler Thromb Vasc Biol 2004;24:684-690.

48 Rehman J, et al: Exercise acutely increases circulating endothelial progenitor cells and monocyte/macrophage-derived angiogenic cells. J Am Coll Cardiol 2004;43:2314-2318.

49 Laufs U, et al: Physical training increases endothelial progenitor cells, inhibits neointima formation, and enhances angiogenesis. Circulation 2004;109:220-226. 
50 Sandri M, et al: Effects of exercise and ischemia on mobilization and functional activation of blood-derived progenitor cells in patients with ischemic syndromes: results of 3 randomized studies. Circulation 2005;111:3391-3399.

51 Palmer TD, Willhoite AR, Gage FH: Vascular niche for adult hippocampal neurogenesis. J Comp Neurol 2000;425:479-494.

52 Segura I, et al: The neurovascular link in health and disease: an update. Trends Mol Med 2009; 15:439-451.

-53 Carmeliet P, Jain RK: Molecular mechanisms and clinical applications of angiogenesis. $\mathrm{Na}$ ture 2011;473:298-307.

54 Leventhal C, et al: Endothelial trophic support of neuronal production and recruitment from the adult mammalian subependyma. Mol Cell Neurosci 1999;13:450-464.

55 Quinones-Hinojosa A, et al: Cellular composition and cytoarchitecture of the adult human subventricular zone: a niche of neural stem cells. J Comp Neurol 2006;494:415-434.

56 Wiltrout $\mathrm{C}$, et al: Repairing brain after stroke: a review on post-ischemic neurogenesis. Neurochem Int 2007;50:1028-1041.

57 Garcia-Verdugo JM, et al: Architecture and cell types of the adult subventricular zone: in search of the stem cells. J Neurobiol 1998;36: 234-248.

58 Ohab JJ, et al: A neurovascular niche for neurogenesis after stroke. J Neurosci 2006;26: 13007-13016.

59 Teng H, et al: Coupling of angiogenesis and neurogenesis in cultured endothelial cells and neural progenitor cells after stroke. J Cereb Blood Flow Metab 2008;28:764-771.

60 Pereira AC, et al: An in vivo correlate of exercise-induced neurogenesis in the adult dentate gyrus. Proc Natl Acad Sci USA 2007;104: 5638-5643.

61 Van der Borght K, et al: Physical exercise leads to rapid adaptations in hippocampal vasculature: temporal dynamics and relationship to cell proliferation and neurogenesis. Hippocampus 2009;19:928-936.

-62 van Praag H, Kempermann G, Gage FH: Running increases cell proliferation and neurogenesis in the adult mouse dentate gyrus. Nat Neurosci 1999;2:266-270.

63 van Praag H, et al: Exercise enhances learning and hippocampal neurogenesis in aged mice. J Neurosci 2005;25:8680-8685.

-64 Seevinck PR, Deddens LH, Dijkhuizen RM: Magnetic resonance imaging of brain angiogenesis after stroke. Angiogenesis 2010;13: 101-111.

65 Aronen HJ, et al: High microvascular blood volume is associated with high glucose uptake and tumor angiogenesis in human gliomas. Clin Cancer Res 2000;6:2189-2200.

-66 Goldman SA, Chen Z: Perivascular instruction of cell genesis and fate in the adult brain. Nat Neurosci 2011;14:1382-1389.

67 Ding $\mathrm{YH}$, et al: Exercise preconditioning ameliorates inflammatory injury in ischemic rats during reperfusion. Acta Neuropathol 2005; 109:237-246.
68 Yang YR, Wang RY, Wang PS: Early and late treadmill training after focal brain ischemia in rats. Neurosci Lett 2003;339:91-94.

69 Ding Y, et al: Exercise pre-conditioning reduces brain damage in ischemic rats that may be associated with regional angiogenesis and cellular overexpression of neurotrophin. Neuroscience 2004;124:583-591.

70 Deplanque D, et al: Prior TIA, lipid-lowering drug use, and physical activity decrease ischemic stroke severity. Neurology 2006;67: 1403-1410.

71 Stroud N, et al: Prestroke physical activity and early functional status after stroke. J Neurol Neurosurg Psychiatry 2009;80:1019-1022.

72 Krarup LH, et al: Prestroke physical activity is associated with severity and long-term outcome from first-ever stroke. Neurology 2008; 71:1313-1318.

73 Lautenschlager NT, Cox K, Kurz AF: Physical activity and mild cognitive impairment and Alzheimer's disease. Curr Neurol Neurosci Rep 2010;10:352-358.

74 Ries ML, et al: Magnetic resonance imaging characterization of brain structure and function in mild cognitive impairment: a review. J Am Geriatr Soc 2008;56:920-934.

75 Hoth KF, et al: Endothelial function and white matter hyperintensities in older adults with cardiovascular disease. Stroke 2007;38:308312.

76 Iadecola C, Gorelick PB: Converging pathogenic mechanisms in vascular and neurodegenerative dementia. Stroke 2003;34:335337.

77 Aarsland D, et al: Is physical activity a potential preventive factor for vascular dementia? A systematic review. Aging Ment Health 2010; 14:386-395.

78 Hamer M, Chida Y: Physical activity and risk of neurodegenerative disease: a systematic review of prospective evidence. Psychol Med 2009;39:3-11.

79 Angevaren M, et al: Physical activity and enhanced fitness to improve cognitive function in older people without known cognitive impairment. Cochrane Database Syst Rev 2008:CD005381.

80 Vaynman S, Gomez-Pinilla F: Revenge of the 'sit': how lifestyle impacts neuronal and cognitive health through molecular systems that interface energy metabolism with neuronal plasticity. J Neurosci Res 2006;84:699-715.

81 Colcombe SJ, et al: Aerobic fitness reduces brain tissue loss in aging humans. J Gerontol A Biol Sci Med Sci 2003;58:176-180.

82 Kleim JA, Cooper NR, VandenBerg PM: Exercise induces angiogenesis but does not alter movement representations within rat motor cortex. Brain Res 2002;934:1-6.

83 McMorris T, et al: A test of the catecholamines hypothesis for an acute exercise-cognition interaction. Pharmacol Biochem Behav 2008;89:106-115.
84 Kubesch S, et al: Aerobic endurance exercise improves executive functions in depressed patients. J Clin Psychiatry 2003;64:10051012.

85 Hillman CH, Snook EM, Jerome GJ: Acute cardiovascular exercise and executive control function. Int J Psychophysiol 2003;48:307314.

86 Kamijo K, et al: The interactive effect of exercise intensity and task difficulty on human cognitive processing. Int J Psychophysiol 2007;65:114-121.

87 Ploughman M, et al: Endurance exercise regimens induce differential effects on brainderived neurotrophic factor, synapsin-I and insulin-like growth factor I after focal ischemia. Neuroscience 2005;136:991-1001.

88 Ma Q: Beneficial effects of moderate voluntary physical exercise and its biological mechanisms on brain health. Neurosci Bull 2008;24:265-270.

89 Flöel A, et al: Physical activity and memory functions: are neurotrophins and cerebral gray matter volume the missing link? Neuroimage 2010;49:2756-2763.

$\$ 90$ Ruscheweyh R, et al: Physical activity and memory functions: an interventional study. Neurobiol Aging 2011;32:1304-1319.

91 Brosse AL, et al: Exercise and the treatment of clinical depression in adults: recent findings and future directions. Sports Med 2002; 32:741-760.

$\$ 92$ Stewart KJ, et al: Are fitness, activity, and fatness associated with health-related quality of life and mood in older persons? J Cardiopulm Rehabil 2003;23:115-121.

$\checkmark 93$ Stoquart-ElSankari S, et al: Aging effects on cerebral blood and cerebrospinal fluid flows. J Cereb Blood Flow Metab 2007;27:15631572.

94 Beason-Held LL, et al: Longitudinal changes in cerebral blood flow in the older hypertensive brain. Stroke 2007;38:1766-1773.

95 Ainslie PN, et al: Elevation in cerebral blood flow velocity with aerobic fitness throughout healthy human ageing. J Physiol 2008;586: 4005-4010.

96 van Beek AH, et al: Cerebral autoregulation: an overview of current concepts and methodology with special focus on the elderly. J Cereb Blood Flow Metab 2008;28:10711085.

97 Abbott RD, et al: Physical activity in older middle-aged men and reduced risk of stroke: the Honolulu Heart Program. Am J Epidemiol 1994;139:881-893.

98 Haheim LL, et al: Risk factors of stroke incidence and mortality. A 12-year follow-up of the Oslo Study. Stroke 1993;24:1484-1489.

$\$ 99 \mathrm{Hu} \mathrm{FB}$, et al: Physical activity and risk of stroke in women. JAMA 2000;283:29612967.

100 Sattelmair JR, et al: Physical activity and risk of stroke in women. Stroke 2010;41:12431250. 
101 Gillum RF, Mussolino ME, Ingram DD: Physical activity and stroke incidence in women and men: the NHANES I Epidemiologic Follow-up Study. Am J Epidemiol 1996;143:860-869.

102 Sacco RL, et al: Leisure-time physical activity and ischemic stroke risk: the Northern Manhattan Stroke Study. Stroke 1998;29: 380-387.

103 Ellekjar H, et al: Physical activity and stroke mortality in women: ten-year follow-up of the Nord-Trondelag Health Survey 19841986. Stroke 2000;31:14-18.

$104 \mathrm{Hu}$ G, et al: Leisure time, occupational, and commuting physical activity and the risk of stroke. Stroke 2005;36:1994-1999.

105 Evenson KR, et al: Physical activity and ischemic stroke risk: the Atherosclerosis Risk in Communities Study. Stroke 1999;30:13331339.
106 Lee I-M, Paffenbarger RS Jr: Physical activity and stroke incidence: the Harvard Alumni Health Study. Stroke 1998;29:2049-2054.

107 Lee IM, et al: Exercise and risk of stroke in male physicians. Stroke 1999;30:1-6.

108 Lee CD, Folsom AR, Blair SN: Physical activity and stroke risk: a meta-analysis. Stroke 2003;34:2475-2481.

109 Wendel-Vos G, et al: Physical activity and stroke. A meta-analysis of observational data. Int J Epidemiol 2004;33:787-798.

110 Lee IM, et al: Exercise and risk of stroke in male physicians. Stroke 1999;30:1-6.

111 Wannamethee G, Shaper AG: Physical activity and stroke in British middle-aged men. BMJ 1992;304:597-601.

112 Aizer A, et al: Relation of vigorous exercise to risk of atrial fibrillation. Am J Cardiol 2009;103:1572-1577.
13 Mozaffarian D, et al: Physical activity and incidence of atrial fibrillation in older adults: the Cardiovascular Health Study. Circulation 2008; $118: 800-807$.

114 Willey JZ, et al: Physical activity and risk of ischemic stroke in the Northern Manhattan Study. Neurology 2009;73:1774-1779.

115 Grau AJ, et al: Association between recent sports activity, sports activity in young adulthood, and stroke. Stroke 2009;40:426-431.

116 Saunders DH, et al: Physical fitness training for stroke patients. Cochrane Database Syst Rev 2009:CD003316.

17 Cheng E, et al: Comparison of secondary prevention care after myocardial infarction and stroke. Cerebrovasc Dis 2006;21:235241.

18 Cornelissen VA, Fagard RH: Effect of resistance training on resting blood pressure: a meta-analysis of randomized controlled trials. J Hypertens 2005;23:251-259. 